\title{
Sea Bottom Classification by Means of Bathymetric LIDAR Data
}

\author{
J. Velasco, I. Molina, E. Martinez, Á. Arquero and J. F. Prieto
}

\begin{abstract}
Light Detection and Ranging (LIDAR) provides high horizontal and vertical resolution of spatial data located in point cloud images, and is increasingly being used in a number of applications and disciplines, which have concentrated on the exploit and manipulation of the data using mainly its three dimensional nature. Bathymetric LIDAR systems and data are mainly focused to map depths in shallow and clear waters with a high degree of accuracy. Additionally, the backscattering produced by the different materials distributed over the bottom surface causes that the returned intensity signal contains important information about the reflection properties of these materials. Processing conveniently these values using a Simplified Radiative Transfer Model, allows the identification of different sea bottom types. This paper presents an original method for the classification of sea bottom by means of information processing extracted from the images generated through LIDAR data. The results are validated using a vector database containing benthic information derived by marine surveys.
\end{abstract}

Keywords - Image processing, bathymetric LIDAR, Radiative Transfer Model, sea bottom classification.

\section{INTRODUCCIÓN}

$\mathrm{E}^{\mathrm{t}}$ L ESTUDIO del fondo marino de aguas poco profundas tiene un gran número de aplicaciones [1], como por ejemplo: el modelado de transporte de sedimentos, la localización de los hábitat marinos, el monitoreo de áreas de distribución de algas, etc. Además, un seguimiento de las características de los fondos marinos, tales como la pendiente, la rugosidad y la cubierta vegetal, puede mejorar la gestión y el mantenimiento económico de los recursos naturales en las zonas costeras. En este sentido y de acuerdo con cuarto informe del Panel Intergubernamental para el Cambio Climático (Intergovernmental Panel on Climate Change, IPCC) [2], el impacto sobre los sistemas costeros podría acentuarse durante los próximos años debido a la subida del nivel medio del mar. Es por esto que se hace imprescindible acceder a una información topográfica de estos sistemas costeros, continuamente actualizada y de alta calidad [3], [4].

Desde hace unos años, esta información venía siendo adquirida mediante técnicas de Teledetección, empleando sensores pasivos (cuya fuente de irradiación es la luz solar) tanto de satélite [5] como cámaras ópticas multiespectrales aerotransportadas [6]. La principal ventaja de estos sistemas residía en su capacidad para escanear simultáneamente vastas áreas de terreno con cubiertas tanto de suelo, como acuosas. Sin embargo, presentan algunos inconvenientes relacionados con las condiciones meteorológicas de registro y ciertas características de turbidez de las aguas, siendo efectiva su utilización en un rango muy limitado de profundidades de las coberturas acuosas. En la década de los años 1970, una nueva generación de sensores activos (emiten su propio haz y actúan como fuente de irradiación controlada) basados en la tecnología LIDAR [7], [8] han superado las deficiencias iniciales encontradas en la adquisición de estos tipos de datos, y además su utilización en combinación con otros [9], [10] [11], procedentes de sensores de alta resolución espacial [12], mejora en gran medida la precisión de los resultados finales en una clasificación temática.

La información en altura y profundidad de zonas costeras puede ser adquirida de manera aerotransportada por medio de dispositivos láser, topográficos y batimétricos, respectivamente [13]. El LIDAR batimétrico supone un tipo de adquisición aerotransportada que penetra en el agua. La técnica ALB (Airborne Laser Bathymetry) es una disciplina relativamente joven y en crecimiento, que depende del estado de desarrollo de otras técnicas en las áreas de ingeniería de láser, óptica, electro-óptica y electrónica. El registro de datos y el procesamiento son digitales, lo que permite flexibilidad en la generación de productos para satisfacer los distintos requisitos de los usuarios y facilita la fusión con datos de otras fuentes de información. Actualmente, esta técnica se encuentra plenamente operativa, $y$ es considerada como la más eficaz para la extracción de información batimétrica en aguas someras, tanto en aguas interiores como en un entorno litoral.

La mayoría de sistemas LIDAR batimétricos recopilan simultáneamente información de la profundidad del agua y la elevación del terreno, y proporciona un elevamiento topográfico de la interfaz tierra-agua. La limitación más importante para estos sistemas es la claridad del agua, lo que limita la máxima profundidad de estudio [14]. Para lograr una óptima precisión en las mediciones de profundidad se deben cumplir unas normas obligatorias estandarizadas. Valores típicos de profundidad que aseguran una buena precisión en aguas limpias es de 50 metros. Como regla general, se puede esperar el éxito en operaciones a profundidades entre 2 y 3 veces la profundidad de Secchi, medida de claridad del agua mediante un ojo humano, de un patrón estándar (disco blanco y negro), situado en el agua desde un barco [15]. Así, ALB permite obtener Modelos Digitales de profundidad o 
Batimétricos (Digital Depth Models, DDM) con un elevado grado de precisión [16].

Adicionalmente, la intensidad de la señal de retorno del LIDAR proporciona una importante información acerca de las propiedades de reflexión de los diferentes tipos de fondos marinos [17], que puede ser procesada convenientemente para obtener sus correspondientes atributos y clasificarlos.

En este trabajo se presenta una metodología efectiva para la clasificación de los fondos marinos por medio de procesamiento de información extraída a partir de de las imágenes generadas a través de los datos LIDAR. El adecuado procesamiento de los valores de la intensidad de la señal de retorno correspondiente a la retrodifusión producida por los diferentes materiales distribuidos sobre el fondo, de acuerdo con sus propiedades de reflexión, utilizando un Modelo Simplificado de Transferencia Radiativa; permite la identificación de los diferentes tipos de fondos. Los resultados fueron validados utilizando una base de datos vector, que contiene información bentónica derivada de estudios marinos.

La organización del trabajo es la siguiente: en la Sección II se describen la zona de estudio así como las características y validación de la información adquirida. La Sección III muestra detalles del procesamiento de los datos LIDAR. La Sección IV muestra un resumen del modelo propuesto para utilizarlo en la sección posterior. La Sección V muestra el caso de estudio donde se especifican metodología y resultados. Finalmente, se pueden encontrar las conclusiones en la Sección VI.

\section{ADQUISICIÓN, CARACTERÍSTICAS Y VALIDACIÓN DE LOS DATOS}

Para este estudio, en el registro de los datos, se ha utilizado un altímetro láser LIDAR aerotransportado. El modelo de láser utilizado es el Hawk Eye MK II, el cual opera simultáneamente [18] en dos bandas espectrales: el láser topográfico en longitudes de onda de $1.064 \mathrm{~nm}$ (localizada en el infrarrojo del espectro electromagnético) que es empleado para la medición altimétrica del suelo terrestre, y un láser hidrográfico que opera a $532 \mathrm{~nm}$ (localizada en el verde del espectro visible electromagnético), el cual permite la medición de las profundidades en aguas someras. La luz infrarroja (sistema láser tradicional) se refleja de vuelta al avión desde la superficie del agua y de la tierra, mientras que el láser verde adicional viaja a través de la columna de agua (Fig. 1). E1 sensor adquiere los datos con una frecuencia de pulsación de $64 \mathrm{kHz}$ en topografía y de $4 \mathrm{kHz}$ en batimetría.

Además del análisis de los dos pulsos distintos, se utiliza la información proporcionada por el sistema de posicionamiento cinemático en tiempo real (Real Time Kinematic, RTK) y el sistema de navegación inercial (Inertial Navigation System, INS), para establecer las profundidades del agua $y$ las elevaciones de la costa.

La toma de datos se ha llevado a cabo durante las primeras semanas de mayo de 2008 en la zona costera denominada "Manga del Mar Menor" (Murcia, España). Esta zona está situada en el sureste de España y abarca una zona costera de aproximadamente $15 \times 6 \mathrm{~km}^{2}$ en dirección norte-sur (Fig. 2).

Como los datos son binarios, pueden ser importados fácilmente por el software de procesado de imagen. Los conjuntos de datos LIDAR, se obtienen en un formato binario estándar denominado LAS que fue creado por la American Society for Photogrammetry and Remote Sensing (ASPRS).

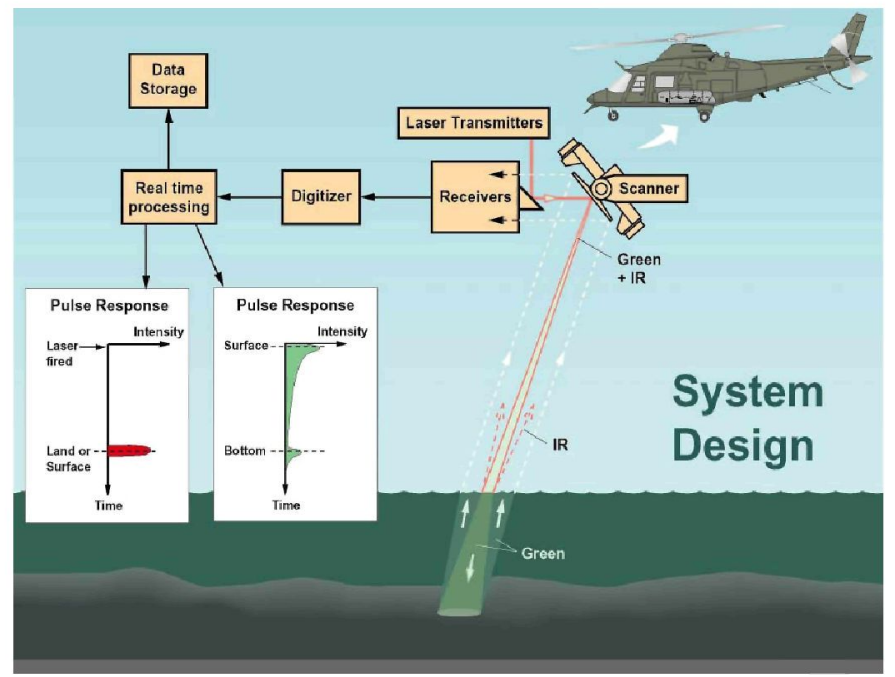

Figura 1. Principio de funcionamiento de una batimetría LIDAR aerotrasportada (Airborne LIDAR Bathymetry, ALB) (@) Admiralty Coastal Surveys AB, http:/www.hydro-international.com/).

Cada archivo LAS contiene metadatos del estudio LIDAR en un bloque de encabezado, seguidos de registros individuales de cada pulso láser registrado. La porción del encabezado de cada archivo LAS contiene información de atributos sobre la topografía LIDAR: extensiones de datos, fecha de vuelo, tiempo de vuelo, cantidad de registros de puntos, cantidad de puntos de retorno, cualquier desplazamiento de datos aplicado y cualquier factor de escala aplicado. Los siguientes atributos de puntos LIDAR se mantienen para cada pulso láser de un archivo LAS: información de ubicación $\mathrm{x}, \mathrm{y}, \mathrm{z}$, marca de tiempo GPS, intensidad, número de retorno, cantidad de retornos, valores de clasificación de puntos, ángulo de escaneo, valores RGB adicionales, dirección de escaneo, borde de línea de vuelo, datos de usuario, identificador de origen de puntos e información de forma de onda. Además del producto básico de profundidades, es precisamente la información de intensidad, la que será utilizada exhaustivamente en este trabajo, para la generación del mapa de intensidades (Fig. 4b).

Las alturas del terreno se validan mediante técnicas Geodésicas GPS. Para este propósito se seleccionaron dos zonas de comprobación en los extremos del área de estudio. Estas zonas se caracterizan por ser completamente planas y facilitan la correlación con los datos LIDAR.

Por otra parte la información batimétrica (profundidad) adquirida se contrasta comparando la nube de puntos con los datos de profundidad adquiridos utilizando un dispositivo de precisión multihaz de alta resolución (Elac Hydrostar 4300), durante las semanas siguientes a la ejecución de la batimetría LIDAR aerotransportada (ALB). Se ha comprobado que ambos conjuntos de datos están dentro de los rangos de precisión estándar. 

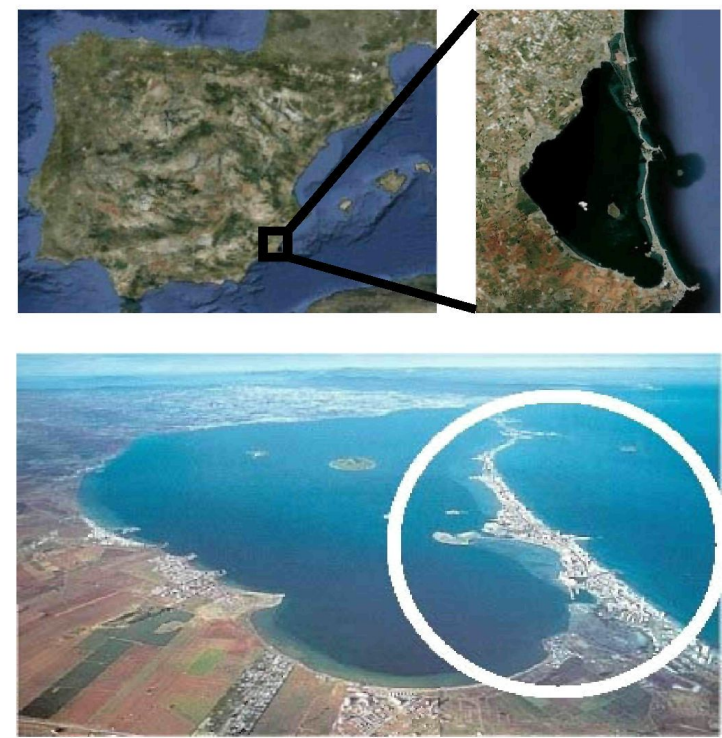

Figura 2. Imágenes de la Manga del Mar Menor (Murcia, España). Tomadas de Google Earth

\section{PROCESAMIENTO DE DATOS LIDAR}

A partir de la información adquirida mediante el sensor aerotransportado y validada posteriormente, se genera el mapa combinado de alturas y profundidades (Fig. 3). Este tipo de productos tiene un amplio abanico de aplicaciones que abarcan desde la gestión propia del litoral hasta los recursos pesqueros en la proximidad de la costa. Además pueden enriquecerse aportando información detallada sobre los tipos de fondos presentes en las aguas someras de la zona, por ello el objetivo perseguido en esta investigación es precisamente clasificarlos.

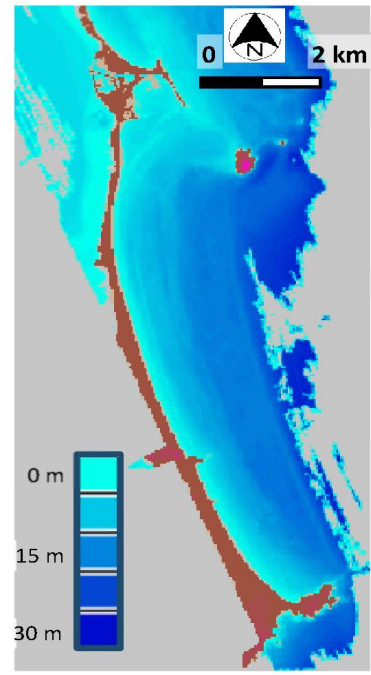

Figura 3. Mapa combinado topográfico y batimétrico del área de estudio.

En dicha imagen, el color gris representa a la zona que no ha sido supervisada adecuadamente, que aparecerá enmascarada en todo el estudio. Los colores marrones corresponden a la cobertura terrestre, y el color azul a la cobertura batimétrica con diferente gradación para diferentes profundidades. Como se observa, en este trabajo (Fig. 3), las profundidades máximas consideradas son aproximadamente de $30 \mathrm{~m}$.

\section{MODElo SimplificAdo DE TRAsferencia RADIATIVA}

Para clasificar los fondos de aguas someras mediante el análisis de los datos procedentes del haz de retorno LIDAR, se utiliza un Modelo Simplificado de Transferencia Radiativa. La intensidad de dicho haz es función tanto de la reflectancia (respuesta espectral relativa de una muestra, a una fuente de irradiación dada) béntica, como de otros parámetros. Este proceso de Teledetección activa se puede modelar mediante un modelo como el mencionado anteriormente, cuya breve descripción se especifica a continuación.

El pulso de láser transmitido desde el sistema LIDAR a través de la columna de agua y reflejado por la superficie hacia al sensor, es atenuado exponencialmente según la profundidad desde la que se refleje. El haz de retorno LIDAR puede ser dividido en tres partes: la superficie del agua, la columna de agua y el retorno del fondo. En este estudio únicamente se considera el último. Wang \& Philpot [19] consideran la expresión física siguiente para describir este proceso:

$P_{R}=P_{T} W \rho e^{\left(-2 k_{s y s} D\right)}$

donde $P_{R}$ es la potencia o intensidad de retorno, $P_{T}$ es la potencia transmitida desde el sensor activo (controlable en el proceso), $W$ combina todos los factores de pérdida del sistema y se considera constante y despreciable para las profundidades consideradas [19], $\rho$ es la reflectancia béntica, $k_{s y s}$ la atenuación específica del agua y $D$ la profundidad del fondo. Tomando logaritmos neperianos en la expresión (1), se obtiene la ecuación (2), lineal con respecto a la profundidad $D$ :

$\ln \left(P_{R}\right)=\ln \left(P_{T} W \rho\right)+-2 k_{s y s} D$

Considerando que $\ln \left(P_{T} W \rho\right)$ es un término constante, el logaritmo neperiano de la potencia recibida es una función lineal de la atenuación del sistema. Para un área específica en la que este coeficiente es también constante, tipos bénticos con reflectancias diferentes describirán líneas paralelas en un gráfico, cuyos ejes cartesianos son $\ln \left(P_{R}\right)$ y $D$. Por lo tanto, la capacidad de detectar cambios de fondo material dependerá de la precisión en la estimación de la profundidad y de la incertidumbre en el coeficiente de atenuación sistema [19].

A continuación, teniendo en cuenta lo comentado anteriormente, relativo a la importante propiedad de estos sistemas, que supone su capacidad de registrar las variaciones de la intensidad de la señal de retorno, que es función del tipo de fondo, propiedades físicas y morfológicas; se puede indicar, que en el caso de una muestra plana como la arena, se generará una fuerte señal de retorno (áreas blancas brillantes de la Fig. 4b), mientras que zonas con vegetación escasamente densa o rocosas, estas producirán un retorno disperso (áreas de color gris oscuro en la Fig. 4b). La Fig. 4 muestra las 
diferencias entre el mapa de profundidades (Fig. 4a) y el mapa de intensidades de la señal de retorno (Fig. 4b), donde se puede observar la independencia existente entre la profundidad y la composición de los materiales del fondo. En ambas imágenes se muestran enmascarada el área correspondiente a la zona no estudiada, en blanco en un caso y en negro en otro, para facilitar su visualización.

Dichas imágenes tienen unas dimensiones de 13156x5531 píxeles y han sido obtenidas directamente desde los archivos originales del registro LIDAR.

\section{Metodologia y Resultados de la Clasificacion}

De acuerdo con el Modelo Simplificado de Trasferencia Radiativa propuesto, se representa en un espacio de características (Fig. 5), la información procedente de la imagen de intensidades y la de la imagen de profundidades.

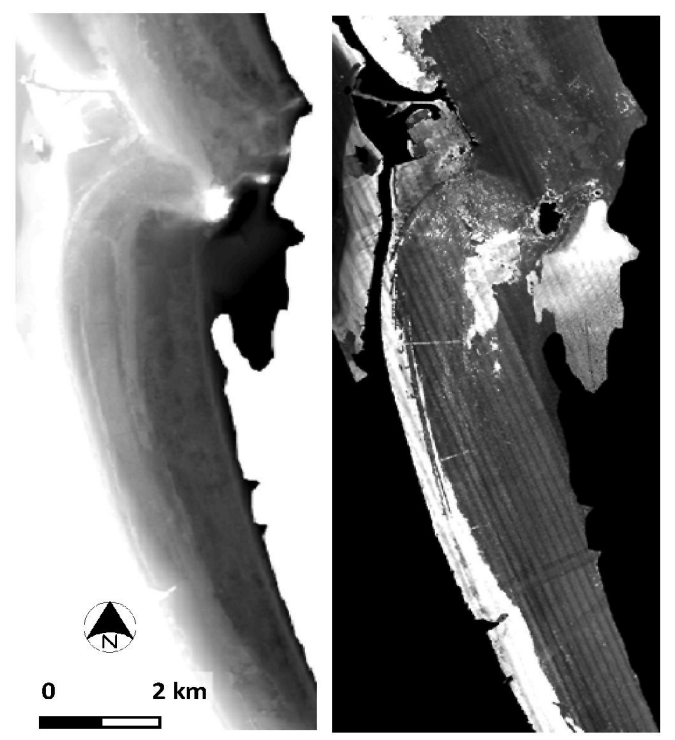

Figura 4. A la izquierda, a) Imagen de profundidades y a la derecha, b) Imagen de intensidades de la zona de estudio.

Se sitúan como variables del dispersograma (Fig. 5a), el logaritmo neperiano de las intensidades de retorno, $\ln \left(P_{R}\right)$, cuyo origen es la imagen de la Fig. $4 \mathrm{~b}$ y las profundidades, $D$, obtenidas de la imagen de la Fig. 4a. El dispersograma está codificado mediante colores, de acuerdo con la mayor (rojo) o menor (magenta) población de píxeles $(N)$ en cada punto del diagrama 2D. En la Fig. $5 b$ se representa el espacio de características 3D, incluyendo el número de integrantes de cada población.

A la imagen multibanda generada con la banda $\ln \left(\mathrm{P}_{\mathrm{R}}\right)$ y con la banda de profundidades (D), se le aplica un método de clasificación, basado en un algoritmo de agrupamiento (clustering) fundamentado en el procedimiento 'Kmeans' supervisado (Fig. 6) mediante la consulta con la base de datos vectorial ESPACE [20], y se obtiene como resultado la imagen clasificada de la Fig. 7a.

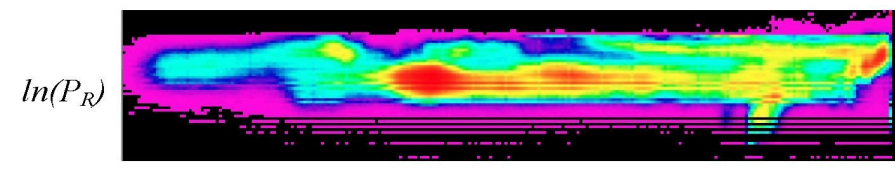

$D$

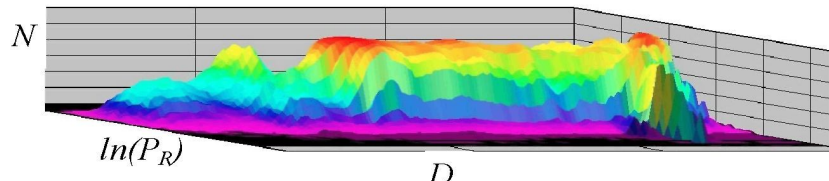

$D$

Figura 5. Superior, a) Dispersograma $\ln \left(P_{R}\right)$ frente a $D$. Inferior, b) Espacio de características $3 \mathrm{D}$

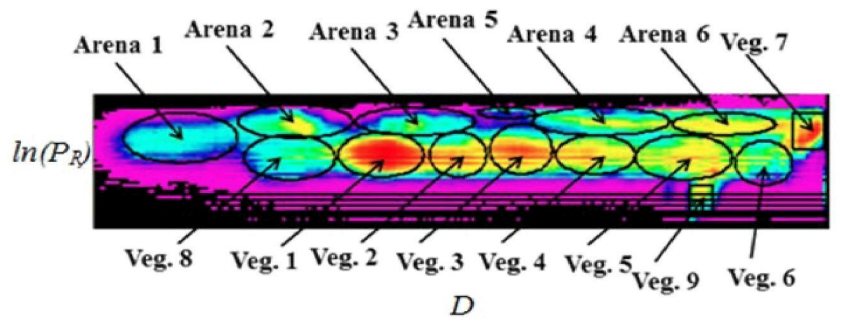

Figura 6. Dispersograma $\ln \left(P_{R}\right)$ frente a $D$, con los agrupamientos supervisados.

Los resultados de la clasificación se muestran en la Tabla I, donde se recopila el porcentaje de píxeles asignados a cada clase y los valores estadísticos de la desviación estándar en las dos bandas (imágenes) que sor ${ }_{D}$ ilizadas para la clasificación.

TABLA I. CARACTERÍSTICAS DE LAS CLASES OBTENIDAS

\begin{tabular}{|c|c|c|c|}
\hline \multirow[b]{2}{*}{ Clase } & \multirow[b]{2}{*}{$\begin{array}{c}\text { POBLACIÓN } \\
(\%)\end{array}$} & \multicolumn{2}{|c|}{ DESVIACIÓN ESTÁNDAR (\%) } \\
\hline & & $\begin{array}{c}\text { BANDA } \\
\ln \left(\mathrm{P}_{\mathrm{R}}\right)\end{array}$ & $\begin{array}{c}\text { BANDA } \\
\text { D }\end{array}$ \\
\hline ARENA 1 & 4,7 & 17,1 & 24,8 \\
\hline ARENA 2 & 4,3 & 11,5 & 13,4 \\
\hline ARENA 3 & 5,1 & 10,1 & 13,1 \\
\hline ARENA 4 & 3,7 & 9,0 & 14,6 \\
\hline ARENA 5 & 2,9 & 10,4 & 13,2 \\
\hline ARENA 6 & 5,1 & 14,0 & 11,0 \\
\hline VEGETACIÓN 1 & 25,4 & 9,8 & 14,2 \\
\hline VEGETACIÓN 2 & 13,5 & 9,3 & 17,2 \\
\hline VEGETACIÓN 3 & 7,6 & 9,1 & 14,3 \\
\hline VEGETACIÓN 4 & 4,0 & 9,3 & 14,0 \\
\hline VEGETACIÓN 5 & 6,4 & 8,6 & 14,6 \\
\hline VEGETACIÓN 6 & 4,3 & 13,7 & 13,4 \\
\hline VEGETACIÓN 7 & 7,7 & 16,6 & 10,8 \\
\hline VEGETACIÓN 8 & 3,6 & 14,9 & 18,1 \\
\hline VEGETACIÓN 9 & 1,6 & 9,8 & 15,1 \\
\hline
\end{tabular}

Se puede considerar que los valores que presentan las desviaciones son adecuados.

Este resultado muestra la capacidad de los datos ALB para discriminar tipos de fondos con el método ensayado, combinación de un modelo de Transferencia Radiativa y un algoritmo de agrupamiento (clustering), en dónde 15 tipos distintos de fondos han sido eficazmente categorizados. Es posible contrastar esta afirmación gracias a la información suministrada por la base de datos generada durante la ejecución del Proyecto ESPACE, en donde se extrajeron intensiva y extensivamente muestras de fondos con el fin de generar una base de datos espacial de estos mismos, los cuales están representados por sus respectivos polígonos. 

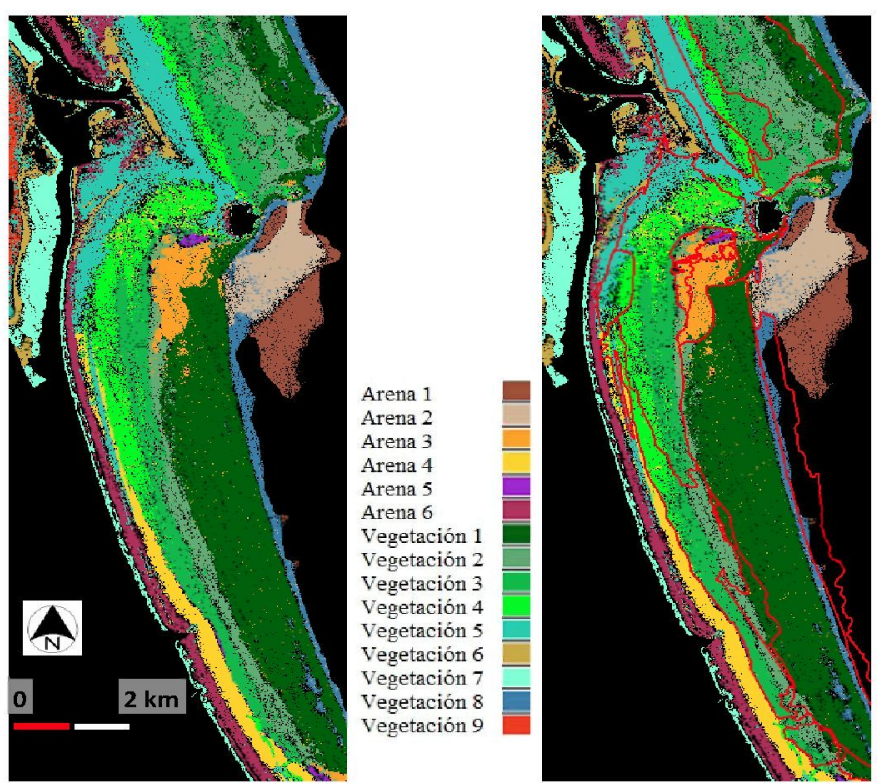

Figura 7. A la izquierda, a) Imagen de intensidades clasificada y a la derecha b) Imagen de intensidades clasificada, con los segmentos SPACE incorporados.

Así pues, la distribución espacial de las clases se valida con los segmentos en formato vector de dicha base de datos, que se incorporan a la imagen clasificada, destacándose mediante bordes de color rojo y que se muestra en la Fig. 7b. Puede apreciarse una perfecta coincidencia entre las fronteras que delimitan las categorías de arenas y de vegetación de fondo, así como entre algunas clases de vegetación. Igualmente, la forma y tipo de una clase concreta de arenas en la parte central de la zona de estudio (Arena 3), coincide perfectamente con los de la base de datos, tal como se observa en la Fig. 7b. Sería conveniente disponer de información adicional que permitiera contrastar otras categorías clasificadas durante este estudio. No obstante, éstas no figuran en la base de datos de referencia, por lo que resulta imposible una comparativa más pormenorizada, quedando esta labor para futuras investigaciones.

\section{CONCLUSIONES.}

Además del gran potencial que tienen datos ALB para la obtención de información batimétrica, en este trabajo, se pone de manifiesto la utilidad de éstos para la clasificación de fondos marinos en un entorno litoral de aguas someras. Para ello se ha utilizado un Modelo Simplificado de Trasferencia Radiativa combinándolo con un procedimiento de agrupamiento (clustering). Esta metodología bajo las condiciones experimentales propias de la zona de estudio, ha permitido discriminar 15 categorías de fondos. De esta forma, los valores de la señal de intensidad de retorno, junto con los datos de profundidad derivados del mismo sensor, se han mostrado muy útiles para caracterizar los tipos de fondos marinos, puesto que las distribuciones espaciales temáticas han resultado ser de calidad adecuada según se ha validado con la información contenida en la base de datos del proyecto ESPACE.

\section{REFERENCIAS}

[1] C. K. Wang and W. D. Philpot, "Using SHOALS LIDAR System to Detect Bottom Material Change", in Proc. IEEE International Geoscience and Remote Sensing Symposium. IGARSS'02, vol. 5, pp. $2690-2692,2002$.

[2] http://www.ipcc.ch/publications_and_data/ar4/wg $1 / \mathrm{en} / \mathrm{ch} 5 . \mathrm{html}$

[3] J. M. Wozencraft, "Complete coastal mapping with airborne lidar", in Proc. OCEANS '02 MTS/IEEE, vol. 2,pp. 1194 - 1198, 2002.

[4] R. Gens, "Remote sensing of coastlines: detection, extraction and monitoring". International Journal of Remote Sensing, 7, pp. 1819-1836, 2010.

[5] Y. J. Kaufman, B. C. Gao and C. O. Davis, "Remote sensing of suspended sediments and shallow coastal waters", IEEE Transactions on Geoscience and Remote Sensing, vol. 41, 3, pp. 559-566, 2003.

[6] P.J. Mumbya, E.P. Greenb, A.J. Edwardsb and C.D. Clarka, "The costeffectiveness of remote sensing for tropical coastal resources assessment and management", Journal of Environmental Management, vol. 55, 3, pp. 157-166, 1999 .

[7] F. Ackermann, "Airborne laser scanning - present status and future expectations". ISPRS Journal of Photogrammetry and Remote Sensing, vol. 54,2 , pp. $64-67,1999$.

[8] E.P. Baltsavias, "Airborne laser scanning: basic relations and formulas". ISPRS Journal of Photogrammetry and Remote Sensing, vol. 54, 2, pp. 199-214, 1999.

[9] J. M. Wozencraft, J. Yong Park and G. Tuell, "Seafloor and Land Cover Classification through Airborne Lidar and Hyperspectral Data Fusion", in Proc. IEEE International Geoscience and Remote Sensing Symposium. IGARSS'08, vol. 2, pp. II-77 - II-80, 2008.

[10] A. F. Elaksher, "Fusion of hyperspectral images and lidar-based dems for coastal mapping". Optics and Lasers in Engineering, vol. 46, 7, pp. 493-498, 2008.

[11] A. Arquero and E. Martinez. "Analysis of Thematic Classified Aerial Images Trough Multispectral and LIDAR Data". IEEE Latin America Transactions, vol. 9, no. 1, pp. 1-7, 2011.

[12] P.R. Marpu, M. Dalla Mura, J.A. Benediktsson and L. Bruzzone, "Classification of Remote Sensing Optical and LiDAR Data Using Extended Attribute Profiles". IEEE Journal of Selected Topies in Signal Processing, vol. 6, no. 7, pp.856-865, 2012.

[13] N. D. Quadros, P.A. Collier and C.S. Fraser. "Integration of Bathymetric and Topographic LIDAR: A preliminary investigation", The International Archives of the Photogrammetry, Remote Sensing and Spatial Information Sciences., vol. XXXVII, Part B8, pp. 1299-1304, 2008.

[14] G.C. Guenther, and L.R. Goodman "Laser applications for near-shore nautical charting", in Proc. SPIE Ocean Optics V, vol. 160, pp. 174-183, 1978.

[15] J. E. Tyler, "The Secchi Disc", Limnology and Oceanography, 13, pp. 1$6,1968$.

[16] A. Collin, A. Cotting, B. Long, P. Archambault, P. Kuus, J. Clarke et al. "Statistical Classification Methodology of SHOALS 3000 Backscatter to Mapping Coastal Benthic Habitats", in Proc. IEEE International Geoscience and Remote Sensing Symposium, IGARSS 2007, Barcelona, Spain, pp. 3178-3181, 2007

[17] G. Tuell, V. Ramnath, J. Feygels, J. Aitken and Y. Kopelivich "Fusion of SHOALS Bathymetric LIDAR and Passive Spectral Data for Shallow Water Rapid Environmental Assessment", in Proc. MTS/IEEE Oceans 2005-Europe, Brest, France, pp. 1046-1051, 2005.

[18] G. Ghust, M. Grande, R. Moncho and I. Galparsoro, "Capacidades del LIDAR Batimétrico HAWK EYE MK II", Teledetección: Agua y desarrollo sostenible, pp. 493-496, 2009.

[19] C.K. Wang and W.D. Philpot, "Using Airborne Bathymetric LIDAR to Detect Bottom Type Variation in Shallow Waters. Remote Sensing of Environment, 106, pp.123-135, 2007.

[20] http://www.ieo.es/ESPACE/descripcion_ESPACE.htm 